Nagwa Amr Lachine ${ }^{1}$, Pacint Elsayed Moez ${ }^{2}$, Kamel Hemida Rohoma ${ }^{1}$, Eiman Mahmoud Abd El Latif ${ }^{3}$, Heba Sadek Kassab ${ }^{1}$

${ }^{1}$ Internal Medicine Department (Unit of Diabetes and Metabolism), Faculty of Medicine, Alexandria University, Alexandria, Egypt

${ }^{2}$ Clinical and Chemical Pathology Department, Faculty of Medicine, Alexandria University, Alexandria, Egypt

${ }^{3}$ Ophthalmology Department, Faculty of Medicine, Alexandria University, Alexandria, Egypt

\title{
Relation between early stages of diabetic retinopathy and early stages of diabetic kidney disease in patients with type 2 diabetes in Egypt
}

\section{ABSTRACT}

Background. Diabetes mellitus is a pandemic disease. Type 2 diabetes (T2DM) is the most common type. Diabetic retinopathy (DR) and diabetic kidney disease (DKD) are disabling chronic complications. The relation between both is not yet well-established in T2DM. Egypt is considered one of the top ten countries regarding the prevalence of diabetes that makes diabetes and its complications a major health problem. This encouraged us to conduct this research.

Materials and methods. The study included 79 patients with T2DM divided into two groups according to the presence of retinopathy. Both groups were subdivided according to urinary albumin to creatinine ratio (UACR) into normoalbuminuric and albuminuric subgroups. Retinopathy group was further subdivided according to severity of retinopathy into mild, moderate and severe non-proliferative DR (NPDR). Statistical analysis was done and relation between the severity of retinopathy and UACR was studied.

Address for correspondence:

Heba Sadek Kassab

Unit of Diabetes \& Metabolism, Department of Internal Medicine

Faculty of Medicine, Alexandria University

17 Champollion Street, El Messallah, Alexandria, Egypt

postcode 21131

Phone: +20 1005536874

e-mail: hebakassab_dm@yahoo.com

Clinical Diabetology 2019, 8, 3, 154-160

DOI: $10.5603 /$ DK.2019.0010

Received: 15.01.2019

Accepted: 26.02.2019
Results. Patients with retinopathy had significantly higher diabetes duration and UACR than non retinopathy group. Also in subgroups of normoalbuminuria and albuminuria, retinopathy group was significantly higher regarding the same parameters. On subdividing the retinopathy group according to severity, severe NPDR group had significantly higher UACR. The severity of DR was significantly positively correlated with UACR. Conclusions. The present study identified a significantly positive correlation between early stages of DR and UACR in patients with T2DM in Egypt. Not all cases of DR had DKD especially in early stages and also not all cases of DKD are associated with the presence of DR in T2DM. (Clin Diabetol 2019; 8, 3: 154-160)

Key words: diabetic retinopathy, diabetic kidney disease, type 2 diabetes

\section{Introduction}

Diabetes mellitus (DM) is considered a global health problem due to its high prevalence and the high incidence of its chronic complications. It is considered the leading cause of blindness, end stage renal disease (ESRD) and lower limb amputation worldwide [1]. According to recent statistics, the Middle East is considered an emerging hot spot in developing diabetes. Egypt is ranked as one of the top ten countries in the prevalence of diabetes (16.7\% of the adult Egyptian population) [2].

Diabetic retinopathy (DR) is one of the major chronic microvascular complications of diabetes. 
Worldwide, the prevalence of DR among people with diabetes is about one third [3]. The duration of diabetes and the level of glycemic control are strongly related to DR. Moreover, chronic hyperglycemia, diabetic kidney disease (DKD), hypertension and dyslipidemia increase the risk of DR [4].

Diabetic kidney disease is another disabling microvascular complication of diabetes. The Diagnosis of DKD is based on the presence of albuminuria and measurement of estimated glomerular filtration rate (eGFR). The pathogenesis of microvascular complications is multifactorial and difficult to understand [5].

The relation between DR and DKD is well-established in type 1 diabetes (T1DM). In type 2 diabetes (T2DM), this association is less clear especially in early stages [6]. The prevalence of DR may reach $63 \%$ in patients with T2DM with proteinuria [7]. The presence of DKD in the absence of DR should suspect a nondiabetic renal disease [8]. This study was conducted to study the relation between early stages of DR and early stages of DKD in T2DM in Egypt.

\section{Materials and methods}

This cross sectional study was conducted on 79 patients with T2DM recruited from the diabetes outpatient clinic of Alexandria Main University Hospital, Faculty of Medicine, Alexandria University, Alexandria, Egypt. Exclusion criteria were: urinary tract infection, estimated glomerular filtration rate (eGFR) less than $60 \mathrm{ml} / \mathrm{min}$, patients with severely increased urinary albumin excretion (UACR > $300 \mathrm{mg} / \mathrm{g}$ ), pregnancy and lactation.

This work was done in accordance with the Ethical Principles for Medical Research Involving Human Subjects outlined in the Helsinki Declaration in 1975 (revised in 2008). An approval was obtained from ethics committee of Faculty of Medicine, Alexandria University. Each participant had signed a written informed consent before participating in the study.

Patients were subjected to full history taking including history of smoking, hypertension, dislipidemia and diabetes duration. Complete physical examination was performed including blood pressure measurement, weight, height, body mass index (BMI) that was calculated as body weight [kg] divided by body height squared $\left[\mathrm{m}^{2}\right]$ and waist circumference (WC) that was measured from the midpoint between highest point of the iliac crest and lowest point of the costal margin at the end of normal expiration according to the WHO recommendation. Screening for diabetic sensorimotor polyneuropathy was done using: The $10 \mathrm{~g}$ monofilament using Semmes-Weinstein 5.07 (10 g) monofilament [9], vibration sense: using a neurothesiometer, the methods were based on the International Working Group on the Diabetic Foot of the IDF [10] and the ankle reflex was also examined.

Fundus examination was done in ophthalmology outpatient clinic of Alexandria Main University Hospital using slit lamp biomicroscope plus fundus lens by the same efficient ophthalmology consultant for all cases.

Eight $\mathrm{ml}$ of blood were drawn from the anticubital vein for each patient and a spot of urine sample was taken for complete urine analysis and measurement of urinary albumin to creatinine ratio (UACR). Albuminuria was confirmed by being positive in two of three specimens of UACR collected within a 3 to 6-month period. Each blood sample was divided between $\mathrm{K}_{2}$-EDTA vacutainer tubes for glycated heamoglobin $\left(\mathrm{HbA}_{1 \mathrm{c}}\right)$ and a clot activator serum vacutainer tube for chemistry tests. Routine laboratory tests were done as follows: $\mathrm{HbA}_{1 \mathrm{c}^{\prime}}$ fasting plasma glucose, blood urea, serum creatinine and measurement of estimated GFR using The Chronic Kidney Disease Epidemiology Collaboration (CKD-EPI) equation [11], total serum cholesterol, high density lipoprotein cholesterol (HDL-C), low density lipoprotein cholesterol (LDL-C) and serum triglycerides.

Cases were divided according to the presence of diabetic retinopathy into two groups (Table 1): 49 patients without retinopathy and 30 patients with retinopathy (all had nonproliferative diabetic retinopathy (NPDR) divided according to severity into mild ( $n=12$ ), moderate $(n=13)$ and severe NPDR $(n=5)$ (Table 2$)$.

Each group was further subdivided into two subgroups according to urinary albumin to creatinine ratio (UACR) into normoalbuminuric group (UACR $<30 \mathrm{mg} / \mathrm{g}$ ) and albuminuric group (with moderately increased urinary albumin excretion UACR 30-300 $\mathrm{mg} / \mathrm{g}$ [formerly called microalbuminuria]). Within the group without retinopathy, 26 patients had normoalbuminuria and 23 patients had albuminuria while in the retinopathy group, 13 patients had normoalbuminuria and 17 patients had albuminuria. The base line characteristics of each subgroup are presented in Table 3. Correlations between severity of DR and other parameters were measured (Table 4).

Statistical analysis of the data was done using IBM SPSS software package version 20.0. (Armonk, NY: IBM Corp). Qualitative data were described using number and percent. The Kolmogorov-Smirnov test was used to verify the normality of distribution. Quantitative data were described using range (minimum and maximum), mean, standard deviation and median. Significance of the obtained results was judged at the $5 \%$ level.

The used tests were:

1. Chi-square test: for categorical variables, to compare between different groups. 
2. Fisher's Exact or Monte Carlo correction: correction for chi-square when more than $20 \%$ of the cells have expected count less than 5 .

3. Student $\mathbf{t}$ test: for normally distributed quantitative variables, to compare between two groups.

4. F-test (ANOVA): for normally distributed quantitative variables, to compare between more than two groups, and post hoc test (LSD) for pairwise comparisons.

5. Kruskal-Wallis test: for abnormally distributed quantitative variables, to compare between more than two studied groups and post hoc (Dunn's multiple comparisons test) for pairwise comparisons.

6. Spearman correlation coefficient was used to identify the correlation between the severity of retinopathy and other parameters.

\section{Results}

Among the 79 patients with diabetes included in this study, 49 had no retinopathy and 30 had diabetic retinopathy (all of them had nonprolifirative diabetic retinopathy (NPDR) with different stages). The two groups were comparable regarding different parameters with no statistically significant difference except for few parameters (Table 1).

Regarding duration of diabetes it was highly statistically significantly increased in the retinopathy group than in the no retinopathy group. Regarding peripheral neuropathy assessment (monofilament, ankle reflex and vibration sense (using vibration perception threshold by neurothesiometer)), they were highly significantly different between the 2 groups. Also UACR was highly significantly increased in the retinopathy group than in the group without retinopathy.

Table 1. Comparison between the two studied groups according to different parameters

\begin{tabular}{|c|c|c|c|c|}
\hline & No retinopathy $(n=49)$ & Retinopathy $(n=30)$ & Test of sig. & $\mathbf{P}$ \\
\hline \multicolumn{5}{|l|}{ Gender } \\
\hline Male & $32(65.3 \%)$ & $17(56.7 \%)$ & $\chi^{2}=0.590$ & 0.443 \\
\hline Female & $17(34.7 \%)$ & $13(43.3 \%)$ & & \\
\hline \multicolumn{5}{|l|}{ Age (years) } \\
\hline Median (min.-max.) & $51(30-65)$ & $52.5(38-68)$ & $t=0.902$ & 0.370 \\
\hline Mean \pm SD & $50.2 \pm 8.2$ & $51.9 \pm 8$ & & \\
\hline \multicolumn{5}{|l|}{ Diabetes duration (years) } \\
\hline Median (min.-max.) & $3(0.5-23)$ & $8.5(1-20)$ & $U=342.5^{*}$ & $<0.001 *$ \\
\hline Mean \pm SD & $4.3 \pm 4$ & $8.9 \pm 5.6$ & & \\
\hline \multicolumn{5}{|l|}{ BMI $\left[\mathrm{kg} / \mathrm{m}^{2}\right]$} \\
\hline Median (min.-max.) & $30(20-45)$ & $28.8(21.5-42)$ & $\mathrm{t}=1.230$ & 0.223 \\
\hline Mean \pm SD & $30.7 \pm 6.1$ & $29.1 \pm 4.7$ & & \\
\hline \multicolumn{5}{|l|}{ Waist circumference $[\mathrm{cm}]$} \\
\hline Median (min.-max.) & $100(71-140)$ & $103(82-125)$ & $t=0.756$ & 0.452 \\
\hline Mean \pm SD & $100 \pm 11.9$ & $102 \pm 10.9$ & & \\
\hline \multicolumn{5}{|l|}{ Monofilament } \\
\hline Absent & $1(2 \%)$ & $4(13.3 \%)$ & $\chi^{2}=17.417^{*}$ & $<0.001 *$ \\
\hline Normal & 46 (93.9\%) & $16(53.3 \%)$ & & \\
\hline Decreased & $2(4.1 \%)$ & $10(33.3 \%)$ & & \\
\hline \multicolumn{5}{|l|}{ Vibration (VPT) } \\
\hline Median (min.-max.) & $15(6-50)$ & $33.5(9-100)$ & $U=318.5^{*}$ & $<0.001 *$ \\
\hline Mean \pm SD & $16.8 \pm 9.6$ & $34.1 \pm 21.2$ & & \\
\hline \multicolumn{5}{|l|}{ Ankle reflex } \\
\hline Absent & $15(30.6 \%)$ & $18(60 \%)$ & $\chi^{2}=9.709^{*}$ & $0.008^{*}$ \\
\hline Normal & $29(59.2 \%)$ & $7(23.3 \%)$ & & \\
\hline Reinforcement & $5(10.2 \%)$ & $5(16.7 \%)$ & & \\
\hline \multicolumn{5}{|l|}{$\mathrm{FPG}[\mathrm{mg} / \mathrm{dl}]$} \\
\hline Median (min.-max.) & $150(80-390)$ & $156(102-336)$ & $U=722.0$ & 0.895 \\
\hline Mean \pm SD & $164.3 \pm 58.9$ & $172.6 \pm 66.4$ & & \\
\hline \multicolumn{5}{|l|}{$\mathrm{HbA}_{1 \mathrm{c}}(\%)$} \\
\hline Median (min.-max.) & $8.1(5.6-14.5)$ & $9(6-13.3)$ & $t=1.300$ & 0.197 \\
\hline Mean \pm SD & $8.6 \pm 1.8$ & $9.2 \pm 1.8$ & & \\
\hline
\end{tabular}


Table 1 (cd). Comparison between the two studied groups according to different parameters

\begin{tabular}{|c|c|c|c|c|}
\hline & No retinopathy $(n=49)$ & Retinopathy $(n=30)$ & Test of sig. & $\mathbf{P}$ \\
\hline \multicolumn{5}{|l|}{ Albumin $[\mathrm{g} / \mathrm{dl}]$} \\
\hline Median (min.-max.) & $3.7(2.8-4.8)$ & $3.7(3-4.9)$ & $t=0.172$ & 0.864 \\
\hline Mean \pm SD & $3.7 \pm 0.4$ & $3.7 \pm 0.4$ & & \\
\hline \multicolumn{5}{|l|}{ Creatinine [mg/dl] } \\
\hline Median (min.-max.) & $0.8(0.5-1.2)$ & $0.8(0.5-1.2)$ & $t=0.688$ & 0.494 \\
\hline Mean \pm SD & $0.8 \pm 0.1$ & $0.8 \pm 0.2$ & & \\
\hline \multicolumn{5}{|l|}{ eGFR [EPI] $\mathrm{ml} / \mathrm{min}$} \\
\hline Median (min.-max.) & $96(61-135)$ & $97.8(61-125.8)$ & $t=0.178$ & 0.859 \\
\hline Mean \pm SD & $94.5 \pm 17.4$ & $95.2 \pm 16.6$ & & \\
\hline \multicolumn{5}{|l|}{ Cholesterol [mg/dl] } \\
\hline Median (min.-max.) & $200(113-272)$ & $187(106-285)$ & $t=1.115$ & 0.268 \\
\hline Mean \pm SD & $194 \pm 39$ & $183.6 \pm 42.1$ & & \\
\hline \multicolumn{5}{|l|}{ Triglycerides [mg/dl] } \\
\hline Median (min.-max.) & $162(46-300)$ & $166.5(43-336)$ & $t=0.232$ & 0.818 \\
\hline Mean \pm SD & $159.5 \pm 53.3$ & $163.2 \pm 76.9$ & & \\
\hline \multicolumn{5}{|l|}{$\mathrm{HDL}-\mathrm{C}[\mathrm{mg} / \mathrm{dll}]$} \\
\hline Median (min.-max.) & $45(24-74)$ & $44.5(25-55)$ & $t=1.051$ & 0.297 \\
\hline Mean \pm SD & $46.5 \pm 10$ & $44.2 \pm 8.5$ & & \\
\hline \multicolumn{5}{|l|}{ LDL-C [mg/dl] } \\
\hline Median (min.-max.) & $110(51.8-188)$ & $108(59-201)$ & $\mathrm{t}=0.414$ & 0.680 \\
\hline Mean \pm SD & $113 \pm 36.2$ & $109.7 \pm 32.8$ & & \\
\hline \multicolumn{5}{|l|}{ UACR $[\mathrm{mg} / \mathrm{g}]$} \\
\hline Median (min.-max.) & $20(3.4-91)$ & $91.3(6-298)$ & $U=362.5^{*}$ & $<0.001$ * \\
\hline Mean \pm SD & $31.5 \pm 25.9$ & $96.6 \pm 84.4$ & & \\
\hline
\end{tabular}

$\chi^{2}-$ Chi-square test; $\mathrm{t}-$ Student $\mathrm{t}$-test; $\mathrm{U}-$ Mann-Whitney test; $\mathrm{p}-\mathrm{p}$ value for comparing between the studied groups; ${ }^{*}$ statistically significant at $\mathrm{p} \leq 0.05$

Table 2. Relation between severity retinopathy and different parameters $(n=30)$

\begin{tabular}{|c|c|c|c|c|c|}
\hline & \multicolumn{3}{|c|}{ Severity Retinopathy } & \multirow[t]{2}{*}{ Test of sig. } & \multirow[t]{2}{*}{$\mathbf{p}$} \\
\hline & $\begin{array}{l}\text { Mild NPDR } \\
(n=12)\end{array}$ & $\begin{array}{l}\text { Moderate NPDR } \\
\qquad(n=13)\end{array}$ & $\begin{array}{l}\text { Severe NPDR } \\
\quad(n=5)\end{array}$ & & \\
\hline \multicolumn{6}{|l|}{ Diabetes duration (years) } \\
\hline Median (min.-max.) & $6(1-12)$ & $7(2-20)$ & $10(3-20)$ & $H=2.167$ & 0.338 \\
\hline Mean \pm SD & $6.7 \pm 3.7$ & $9.9 \pm 6.6$ & $11.2 \pm 6.1$ & & \\
\hline \multicolumn{6}{|l|}{ eGFR EPI [ml/min] } \\
\hline Median (min.-max.) & $93(63.5-123)$ & $97(61-125.8)$ & $102(72-110)$ & $F=0.222$ & 0.802 \\
\hline Mean \pm SD & $92.7 \pm 17.3$ & $96.6 \pm 17.6$ & $97.5 \pm 15$ & & \\
\hline \multicolumn{6}{|l|}{ UACR $[\mathrm{mg} / \mathrm{g}]$} \\
\hline Median (min.-max.) & $32.1(6-134)$ & $29.9(27.3-163.5)$ & $270(179.5-298)$ & $H=14.155^{*}$ & 0.001 * \\
\hline Mean \pm SD & $55.7 \pm 47.3$ & $79.2 \pm 59.6$ & $240^{\mathrm{ab}} \pm 55.9$ & & \\
\hline
\end{tabular}

$\mathrm{F}$ - F for ANOVA test; $\mathrm{H}-\mathrm{H}$ for Kruskal-Wallis test. Pairwise comparison bet. each 2 groups was done using post hoc test (Dunn's for multiple comparisons test); $p-p$ value for comparing between different categories; *statistically significant at $p \leq 0.05 ; a-$ significant with mild NPDR; $b-$ significant with moderate NPDR; UACR — urinary albumin to creatinine ratio; NPDR - non-proliferative diabetic retinopathy

Subdividing each group according to UACR into normoalbuminuric group and albuminuric group, the duration of diabetes was significantly higher in both retinopathy subgroups than in both subgroups without retinopathy but not between the albuminuric and normoalbuminuric subgroups within each group (Table 3). Regarding UACR it was significantly higher in the albuminuric group with retinopathy than the 3 other subgroups while there was no statistically significant difference between the 2 normoalbuminuric 
Table 3. Comparison between the four studied subgroups according to different parameters

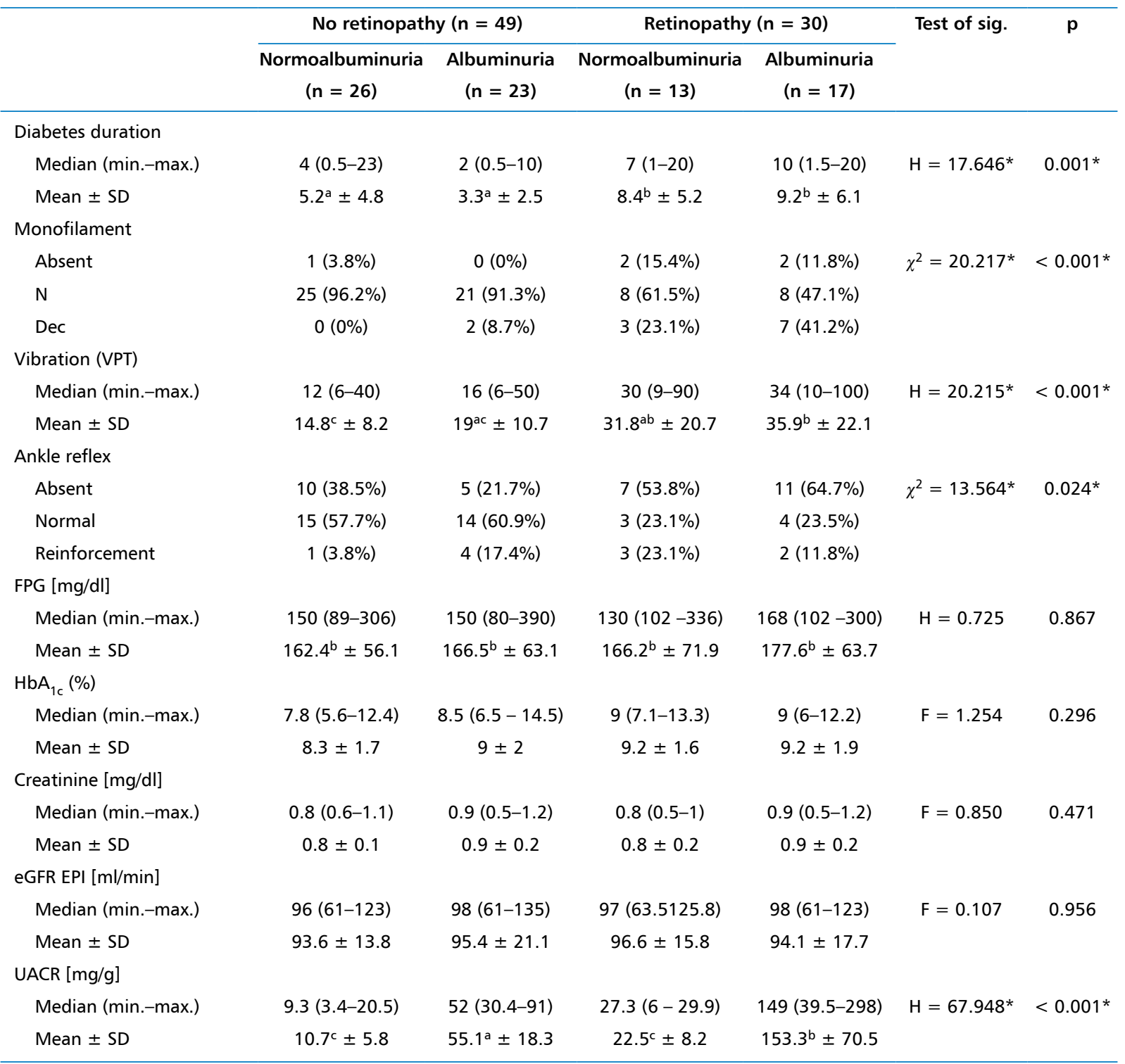

$\chi^{2}-$ Chi-square test; $\mathrm{F}-\mathrm{F}$ for ANOVA test; $\mathrm{H}-\mathrm{H}$ for Kruskal Wallis testi $\mathrm{p}-\mathrm{p}$ value for comparing between the studied groups; *statistically significant at $p \leq 0.05$. Means with common letters are not significant (i.e. means with different letters are significant)

subgroups regarding UACR although the mean value of UACR was higher in the normoalbuminuric group with retinopathy.

Regarding severity of retinopathy (Table 2) the three subgroups were comparable regarding different parameters without statistically significant difference except for UACR that was highly significantly higher in cases of severe NPDR.

On performing correlation between severity of retinopathy and different parameters, it was highly significantly positively correlated with UACR with no significant correlation with other parameters (Table 4, Figure 1).
Table 4. Correlation between severity of retinopathy and different parameters $(n=30)$

\begin{tabular}{lcc}
\hline & \multicolumn{2}{c}{ Severity retinopathy } \\
\cline { 2 - 3 } & $\mathrm{r}_{\mathrm{s}}$ & $\mathbf{p}$ \\
\hline UACR & $0.642^{*}$ & $<0.001^{*}$ \\
eGFR EPI & 0.137 & 0.472 \\
Diabetes duration & 0.273 & 0.144 \\
Systolic blood pressure [mm Hg] & 0.097 & 0.612 \\
Diastolic blood pressure [mm Hg] & -0.034 & 0.858 \\
\hline
\end{tabular}

$r_{s}$ - Spearman coefficient; *statistically significant at $p \leq 0.05$; UACR urinary albumin to creatinine ratio; eGFR - estimated glomerular filtration rate 


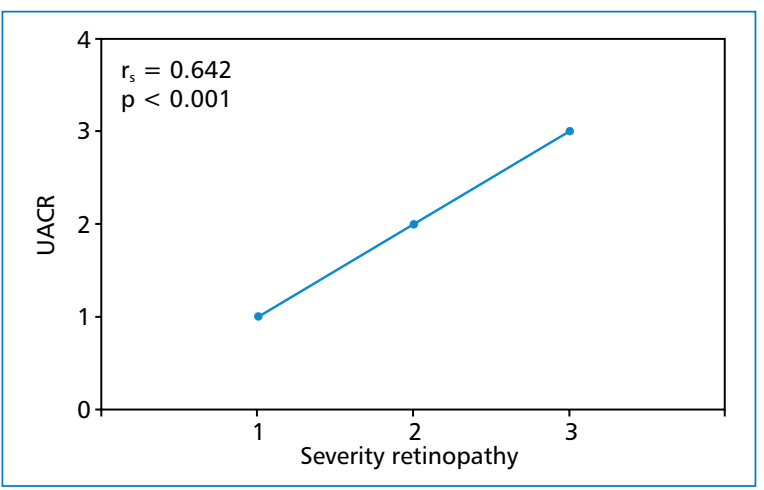

Figure 1. Correlation between severity of retinopathy and UACR $(n=30)$. Severity of retinopathy: 1 - mild NPDR; $2-$ moderate NPDR; 3 - severe NPDR. UACR - urinary albumin to creatinine ratio; NPDR - non-proliferative diabetic retinopathy

\section{Discussion}

Although the association between diabetic retinopathy and DKD is well established in T1DM, it is less evident in T2DM especially in early cases of DKD with moderately increased UACR. To the best of our knowledge, no previous studies clearly discussed the relation between early stages of diabetic retinopathy and early stages of DKD in T2DM in Egypt. That's why we conducted the present study.

In the present study, the patients with retinopathy had significantly higher UACR, significantly longer diabetes duration and significantly higher prevalence of peripheral neuropathy. Otherwise, groups and subgroups were comparable regarding other parameters.

Regarding the severity of DR, severe NPDR cases had significantly higher UACR than the other 2 groups. The severity of retinopathy was highly significantly positively correlated with UACR.

Sanyal et al. [12] studied the association between retinopathy and nephropathy in diabetic patients with advanced renal disease. They concluded that retinopathy without nephropathy is common while nephropathy without retinopathy is rare. However, their patients had had advanced renal disease (ESRD) and this explains the discordance between their results and ours as we studied patients with diabetes with early stages of DKD and DR.

A meta-analysis involving 2012 patients from 26 studies found that DR is useful in screening for or diagnosing DKD in patients with T2DM and renal disease, and they recognized proliferative $D R$ as a highly specific indicator for DN [13]. The present study had similar results but regarding early stages of both complications.

In agreement with the results of the present study, Kotlarsky et al. [14] concluded that the degree of renal impairment is proportional to the degree of eye damage. In addition, they statistically proved the chronological relation between retinopathy and DKD; that the renal injury precedes the retinopathy.

Romero-Aroca et al. [15] studied the relationship between DR and DKD in T2DM and concluded that both UACR and eGFR are important risk factors of DR, although UACR has a better association. The present study revealed similar results regarding the relation between DR and UACR but not regarding eGFR, this may be due to the early stages of the disease included in the present study.

Zhang et al. [16] studied the relationship between DR and the DKD progression in patients with T2DM. They concluded that in patients with T2DM and DKD, DR may predict the renal prognosis. They found a significant association between the severity of glomerular lesions and DR.

Although the pathogenesis of all microvascular complications is similar (polyol pathway, protein kinase C pathway and advanced glycation end products) [17], this difference in the time of the beginning of each complication may be a result of genetic factors determining the susceptibility to DR irrespective to DKD. Many studies reported specific genes associated with or protective against DR [18-21]. Further studies are needed to prove the association between DR and DKD in T2DM with the identification of chronological sequence which occurs first.

\section{Conclusions}

The present study revealed significant relation between the severity of DR and the degree of albuminuria in early stages of DKD in cases with T2DM. Further studies are needed to confirm this relation and to reveal the chronological sequence of diabetic microvascular complications in T2DM.

Not all cases of DR have DKD especially in early stages and also not all cases of DKD are associated with the presence of DR in T2DM. Genetic predisposition may be present.

The study also concluded that patients with DR have significantly longer diabetes duration than patients without DR.

\section{Conflict of interest}

The authors declare no conflict of interest.

\section{Acknowledgement and funding}

This research was funded by Internal Medicine Department (Diabetes Unit) and Department of Chemical \& Clinical Pathology, Faculty of Medicine, Alexandria University, Egypt. 


\section{REFERENCES}

1. American Diabetes Association. Standards of medical care in diabetes 2019. Diabetes Care. 2019; 42(Suppl 1): S1-S2, doi: doi. org/10.2337/dc19-Sint01.

2. Khalil SH, Megallaa MH, Rohoma KH, et al. Prevalence of type 2 diabetes mellitus in a sample of the adult population of Alexandria, Egypt. Diabetes research and clinical practice 2018; 144: 63-73, doi:10.1016/j.diabres.2018.07.025.

3. Yau JWY, Rogers SL, Kawasaki R, et al. Meta-Analysis for Eye Disease (META-EYE) Study Group. Global prevalence and major risk factors of diabetic retinopathy. Diabetes Care. 2012; 35(3): 556-564, doi: 10.2337/dc11-1909, indexed in Pubmed: 22301125.

4. Chew EY, Davis MD, Danis RP, et al. Action to Control Cardiovascular Risk in Diabetes Eye Study Research Group. The effects of medical management on the progression of diabetic retinopathy in persons with type 2 diabetes: the Action to Control Cardiovascular Risk in Diabetes (ACCORD) Eye Study. Ophthalmology. 2014; 121(12): 2443-2451, doi: 10.1016/j.ophtha.2014.07.019, indexed in Pubmed: 25172198.

5. American Diabetes Association. 11. Microvascular Complications and Foot Care: . Diabetes Care. 2019; 42(Suppl 1): S124-S138, doi: 10.2337/dc19-S011, indexed in Pubmed: 30559237.

6. Romero-Aroca P, Mendez-Marin I, Baget-Bernaldiz M, et al. Review of the Relationship between Renal and Retinal Microangiopathy in Diabetes Mellitus Patients. Current Diabetes Reviews. 2010; 6(2): 88-101, doi: 10.2174/157339910790909387.

7. Collins AJ, Foley RN, Gilbertson DT, et al. United States Renal Data System public health surveillance of chronic kidney disease and end-stage renal disease. Kidney Int Suppl (2011). 2015; 5(1): 2-7, doi: 10.1038/kisup.2015.2, indexed in Pubmed: 26097778.

8. Kanauchi M, Kawano T, Uyama H, et al. Discordance between retinopathy and nephropathy in type 2 diabetes. Nephron. 1998; 80(2): 171-174, doi: 10.1159/000045162, indexed in Pubmed: 9736815.

9. Pham $\mathrm{H}$, Armstrong DG, Harvey $\mathrm{C}$, et al. Screening techniques to identify people at high risk for diabetic foot ulceration: a prospective multicenter trial. Diabetes Care. 2000; 23(5): 606-611, indexed in Pubmed: 10834417.

10. Apelqvist J, Bakker K, van Houtum WH, et al. International Working Group on the Diabetic Foot (IWGDF) Editorial Board. Practical guidelines on the management and prevention of the diabetic foot: based upon the International Consensus on the Diabetic Foot (2007) Prepared by the International Working Group on the Diabetic Foot. Diabetes Metab Res Rev. 2008; 24 Suppl 1: S181-S187, doi: $10.1002 / d m r r .848$, indexed in Pubmed: 18442189 .
11. Stevens LA, Schmid CH, Zhang YL, et al. Development and validation of GFR-estimating equations using diabetes, transplant and weight. Nephrol Dial Transplant. 2010; 25(2): 449-457, doi: 10.1093/ndt/gfp510, indexed in Pubmed: 19793928.

12. Sanyal D, Chatterjee S. Advanced Diabetic Nephropathy with "Clean" Eyes: An Extreme Phenotype. Indian J Endocrinol Metab. 2018; 22(2): 274-276, doi: 10.4103/ijem.IJEM_590_17, indexed in Pubmed: 29911044.

13. He F, Xia X, Wu XF, et al. Diabetic retinopathy in predicting diabetic nephropathy in patients with type 2 diabetes and renal disease: a meta-analysis. Diabetologia. 2013; 56(3): 457-466, doi: 10.1007/ /s00125-012-2796-6, indexed in Pubmed: 23232641.

14. Kotlarsky P, Bolotin A, Dorfman K, et al. Link between retinopathy and nephropathy caused by complications of diabetes mellitus type 2. Int Ophthalmol. 2015; 35(1): 59-66, doi: 10.1007/s10792014-0018-6, indexed in Pubmed: 25391917.

15. Romero-Aroca P, Baget-Bernaldiz M, Navarro-Gil R, et al. Glomerular Filtration Rate and/or Ratio of Urine Albumin to Creatinine as Markers for Diabetic Retinopathy: A Ten-Year Follow-Up Study. Journal of Diabetes Research. 2018; 2018: 1-9, doi: 10.1155/2018/5637130.

16. Zhang J, Wang Y, Li Li, et al. Diabetic retinopathy may predict the renal outcomes of patients with diabetic nephropathy. Ren Fail. 2018; 40(1): 243-251, doi: 10.1080/0886022X.2018.1456453, indexed in Pubmed: 29633887.

17. Brownlee M. Biochemistry and molecular cell biology of diabetic complications. Nature. 2001; 414(6865): 813-820, doi: 10.1038/414813a, indexed in Pubmed: 11742414.

18. Hu QR, Huang $L Z, C h e n ~ X L$, et al. Genetic analysis and clinical features of $X$-linked retinoschisis in Chinese patients. Sci Rep. 2017; 7: 44060, doi: 10.1038/srep44060, indexed in Pubmed: 28272453.

19. Abhary S, Hewitt AW, Burdon KP, et al. A systematic meta-analysis of genetic association studies for diabetic retinopathy. Diabetes. 2009; 58(9): 2137-2147, doi: 10.2337/db09-0059, indexed in Pubmed: 19587357.

20. Uthra S, Raman R, Mukesh BN, et al. Diabetic retinopathy: Validation study of ALR2, RAGE, iNOS and TNFB gene variants in a south Indian cohort. Ophthalmic Genet. 2010; 31(4): 244-251, doi: 10.3109/13816810.2010.523037, indexed in Pubmed: 21067489.

21. Cilenšek I, Mankoč S, Petrovič MG, et al. GSTT1 null genotype is a risk factor for diabetic retinopathy in Caucasians with type 2 diabetes, whereas GSTM1 null genotype might confer protection against retinopathy. Dis Markers. 2012; 32(2): 93-99, doi: 10.3233/DMA-2011-0863, indexed in Pubmed: 22377702 